\title{
Be careful what you eat and drink; Coprinic and Kounis syndrome in a patient with the Samter`s triad
}

BY JOAQUÍN VALLE ALONSO, ELISA LOPERA LOPERA, YELDA HERNÁNDEZ MONTES, CARMEN NAVARRO, PEDRO CEBALLOS GARCÍA, JOSÉ TEJEDA

\begin{abstract}
Allergic reactions to chemicals, food products or even insect bites are encountered all over the world with a variety of manifestations. Symptoms range from the development of a minor rash to life threatening anaphylactic reactions. Sometimes, acute coronary syndrome (ACS) can be registered in such patients, which may have a serious impact on the course and management of the allergic reaction. Mushroom poisoning constitutes the main portion of plant toxicities in Spain. Depending on the type of mushroom, the adverse effects range from mild gastrointestinal (GI) symptoms to major cytotoxic effects resulting in organ failure and death. Coprinopsis atramentaria poisoning is rare but with serious consequences. We report a case of a 27 year old patient with a previous history of mild persistent asthma, nasal polyps and aspirin allergy that arrived to the Emergency Department with dizziness and temor after consuming beer at a party. On arrival to the hospital, the patient suddenly collapsed, with an electrocardiogram (ECG) showing ST elevation in the inferior leads and 3rd degree atrioventricular (AV) block accompanied by urticarial-like lesions. After administration of treatment, the patient and ECG abnormalities improved. A detailed medical history showed that the patient had eaten scrambled mushrooms and beer. Expert analysis demonstrated that the combined consumption of Coprinopsis atramentaria and alcoholic beverages produced a disulfiram-like
\end{abstract}


reaction (Coprinic syndrome) and type I Kounis syndrome. No case like this has been previously reported.

Key words: Kounis syndrome, Coreopsis atramentaria, acute coronary syndrome

\section{Case report}

A 27-year-old man, with no cardiovascular risk factors and a history of mild persistent asthma, nasal polyps and aspirin allergy, was transferred with dizziness and vomiting to the hospital after consumption of beer at a party. Approximately 5 minutes after arrival to the hospital, he complained of chest pain, accompanied by nausea and diaphoresis. Urticarial skin lesions appeared, scattered on the trunk and extremities, and syncope ensued with loss of consciousness. Physical examination demonstrated a heart rate of $30 \mathrm{bpm}$ and severe hypotension $(60 / 40$ $\mathrm{mmHg}$ ). An electrocardiogram (ECG) revealed ST elevation of $2 \mathrm{~mm}$ in leads II, III and aVF, with a specular reflection on leads I and aVL, V1-V4 and 3rd degree atrioventricular (AV) block (figure 1). Following administration of oxygen, adrenaline, corticosteroids, antihistamines, clopidrogel and low molecular weight heparin the patient became hemodynamically stable, The patient's blood pressure by this time was $123 / 76 \mathrm{mmHg}$. The rash disappeared completely over the next 2-3 $\mathrm{h}$ and the electrocardiographic findings returned to normal, with an EGG showing normal sinus rhythm (figure 2), with ST segment correction. With the diagnosis of Kounis syndrome the patient was admitted to the intensive care unit (ICU). Echocardiogram showed hypokinesia of the mid-inferior left ventricle (LV). Blood analysis revealed a total Creatine Kinase (CK) fraction of 1,790 U/L (normal: 25150), CK-MB fraction of $256 \mathrm{U} / \mathrm{L}$ (normal: 5-20), and troponin I value of $3.17 \mathrm{ng} / \mathrm{mL}$ (normal: 0.030). Additional blood tests revealed tryptase values of $15.1 \mathrm{~g} / \mathrm{L}$. Heart catheterization revealed the presence of angiographically normal coronary arteries; 72 hours later serum tryptase values had returned to normal (3.23 g/L). A detailed medical history showed that the patient had consumed scrambled mushrooms and 2 beers at a dinner party; the mushrooms had been obtained from a private garden, a sample was brought (figure 3) and identified by an expert as Coprinopsis atramentaria. Five days after discharge, repeat echocardiogram did not reveal any contractility abnormalities or valvular disease, and the ejection fraction was normal. The temporal association of development of chest pain, ECG changes, positive Troponin, inferior wall hypokinesia with anaphylactic

manifestations due to ingestion of mushrooms and then everything normalizing 
together in a few days strongly suggested that the cardiac event was related to the allergic reaction. The patient also had symptoms and signs suggesting coprinic syndrome secondary to Coprinopsis atramentaria, including facial erythrosis, hypotension and trembling of the extremities. During allergy screening tests, it was found that the patient was allergic to contact, drugs and food. The patient was advised to avoid these allergens and use topical steroids, bilastin and montelukast. Up until the current date, the patient has been doing well.

\section{Discussion}

Mushroom poisoning occurs frequently, but serious toxicity is uncommon. There are 12 groups of identified mushroom toxins with 14 described clinical syndromes. Defining which clinical syndrome predominates, initiating general supportive care, and administering any specific treatments for that syndrome are the key steps in the recognition and management of mushroom poisoning. $(1,2)$ The toxin coprine, when ingested before consumption of ethanol, results in a disulfiramlike reaction. $(1,3,4)$ Coprinopsis atramentaria, the "inky cap" mushroom, and related species are most commonly associated with this syndrome. Previously known as Coprinopsis atramentaria, it is the second best known ink cap and previous member of the genus Coprinus after C. comatus. It is a widespread and common fungus found throughout the northern hemisphere, arises in small clumps from spring until early winter, commonly in urban and disturbed habitats such as vacant lots and lawns, as well as grassy areas. The grey-brown cap is initially bell-shaped before opening, after which, it flattens and disintegrates. Coprine is heat-stable, so boiling or cooking the mushrooms does not prevent toxicity. The metabolites of coprine, including 1-aminocyclopropanol and cyclopropanone hydrate, irreversibly inhibit aldehyde dehydrogenase. $(1,3,4)$ This effect causes a marked elevation of blood aldehyde when ethanol is ingested several hours to seven days after Coprinus mushroom consumption. However, if alcohol is ingested at the same time as coprine-containing mushrooms, toxicity often does not occur or is lessened because the metabolism of coprine is slower than the normal hepatic elimination of ethanol. Clinical effects usually occur within two hours of ethanol ingestion and include; headache, flushing of the face, neck, and trunk, Nausea and vomiting, tachycardia, palpitations, chest pain, dyspnea, marked apprehensiveness, rarely, hypotension, cardiac dysrhythmia, confusion, and/or coma. Treatment of toxicity is supportive. Clinical findings are 
self-limited and typically resolve after three to six hours, although, they can rarely persist as long as 24 hours. (3) Alcohol intolerance can also be precipitated by mushroom species other than Coprinopsis atramentaria (eg, Lepiota aspera). Although the pathophysiology of intolerance and toxicity is not well characterized, the treatment is the same. (5)

The first report of myocardial infarction during an allergic reaction (to penicillin) was published in 1950. (6) Kounis and Zarvas (7) introduced the concept and nomenclature of the "syndrome of allergic angina" in 1991, and 5 years later (8) they described the "syndrome of allergic myocardial infarction". Today, Kounis syndrome is a recognized cause of acute coronary syndrome and many etiologies have been reported, $(9,10)$ including drugs (antibiotics, analgesics, antineoplastics, contrast media, corticosteroids, intravenous anesthetics, non-steroidal antiinflammatory drugs, skin disinfectants, thrombolytics, anticoagulants), various conditions (angioedema, bronchial asthma, rhinitis, nasal polyp, urticaria, food allergy, exercise-induced allergy, mastocytosis, serum sickness), environmental exposure (stings of ants, bees, wasps and jellyfish, grass cuttings, millet allergy, poisoning, latex contact, eating shellfish, viper venom poisoning, anisakis). Until our report, no case had been reported related to mushroom poisoning. Many experts believe that Kounis Syndrome is not a rare disease but a 'rarely diagnosed' disease.

Three variants of Kounis syndrome have been described so far. (11) The type I variant (coronary spasm), which might represent a manifestation of endothelial dysfunction or microvascular angina, includes patients with normal coronary arteries without predisposing factors for coronary artery disease in whom the acute release of inflammatory mediators can induce either coronary artery spasm without increase of cardiac enzymes and troponins or coronary artery spasm progressing to acute myocardial infarction with raised cardiac enzymes and troponins. The type II variant (coronary thrombosis) includes patients with culprit but quiescent pre-existing atheromatous disease in whom the acute release of inflammatory mediators can induce either coronary artery spasm with normal cardiac enzymes and troponins, plaque erosion or rupture, manifesting as acute myocardial infarction. Recently a 3rd variant has been suggested including patients with coronary artery stent thrombosis in whom eosinophils and mast cells were isolated in the thrombus. (12) The diagnosis is primarily clinical and is based on symptoms and signs suggestive of an acute allergic reaction and acute coronary event coincident in time. The patient can present as an acute coronary 
syndrome manifesting as anginal chest pain, nausea and diaphoresis and simultaneously typical symptoms of an anaphylactic reactions in the context of contact with known allergens including hypotension, skin symptoms (rash, urticaria, angioedema), breathing (dyspnoea, wheezing, hoarseness, stridor) and/or gastrointestinal (abdominal pain, nausea, vomiting).

The treatment of these allergic angina pectoris syndromes involves managingboth the acute coronary syndrome and the allergic syndrome simultaneously.

Cevik et al. suggested treating the anaphylactic or anaphylactoid reaction with epinephrine, corticosteroids, antihistamines (both H1- and H2-blockers), fluid resuscitation, and oxygen. (13) During the allergic reaction, if the patient develops chest pain and allergic angina pectoris or if myocardial infarction is suspected, emergent catheterization should be considered for both diagnostic and therapeutic reasons. Without angiographic evidence or a prior history of an allergic angina-type syndrome, it is difficult to start treating someone for this disease in the emergency department. Once vasospasm is diagnosed through cardiac catheterization, vasodilator drugs such as intracoronary nitrates and calcium channel blockers are recommended. Revascularization or antithrombotics may be appropriate in type II variants. If the culprit allergen is known, the implicated causal factor should be strictly avoided. Along with epinephrine, long-term use of mast cell stabilizers, such as cromolyn sodium or nedocromil sodium, and antihistamines may be considered.

The patient had a previous recognized history of mild persistent asthma, nasal polyps and aspirin allergy consistent with the Samter's triad (asthma, aspirin sensitivity, and nasal polyps). The term Samter's triad has become popular. Several cases of Kounis syndrome in patients with Samter's triad have been reported; whether there is a direct relationship in the current case should be further investigated. In this patient there is a rare combination of mushroom poisoning syndrome (coprinic syndrome) and Kounis syndrome. When there is a young individual with no predisposing factors for atherosclerosis, and an apparent coronary lesion, with or without ECG and biochemical markers of infarction, the possibility of Kounis syndrome should always be kept in mind.

Figure 1. Electrocardiogram on arrival showing ST elevation of $2 \mathrm{~mm}$ in leads II, III and aVF, with a specular reflection on leads I and aVL, V1-V4 and 3rd degree atrioventricular (AV) block. 


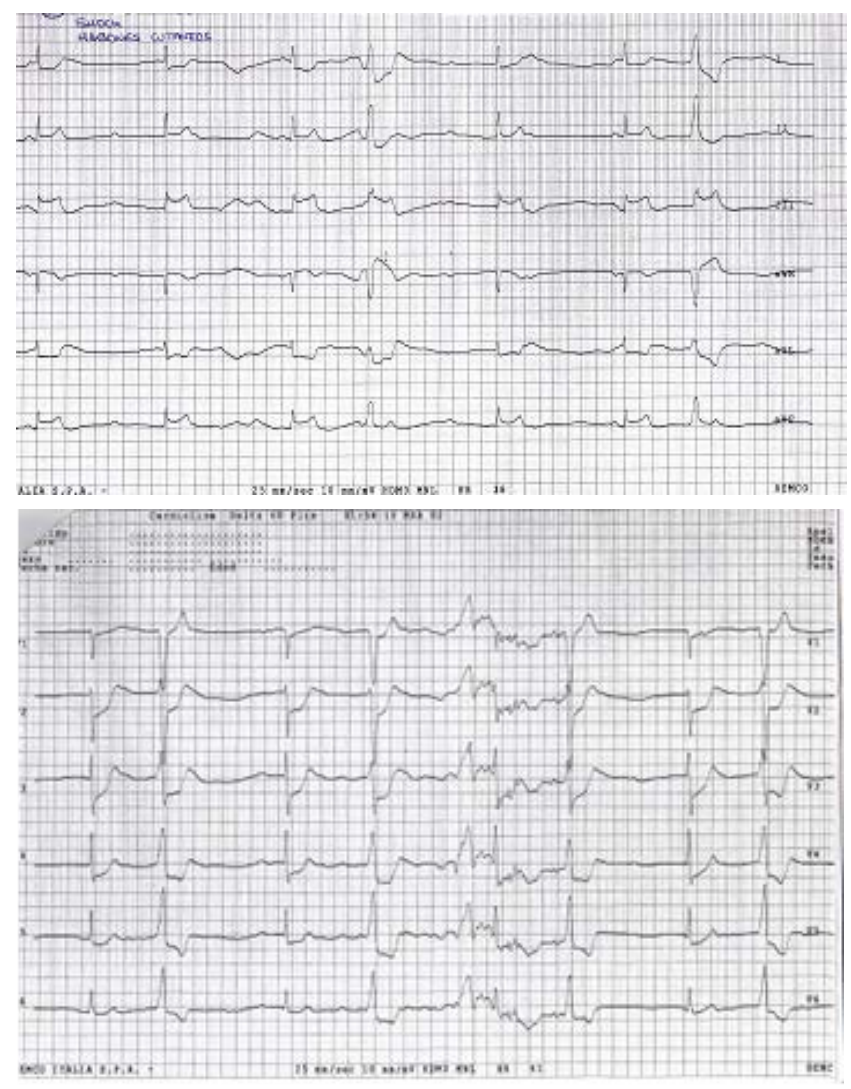

Figure 2. Electrocardiogram showing normal sinus rhythm with ST segment correction after administration of treatment.

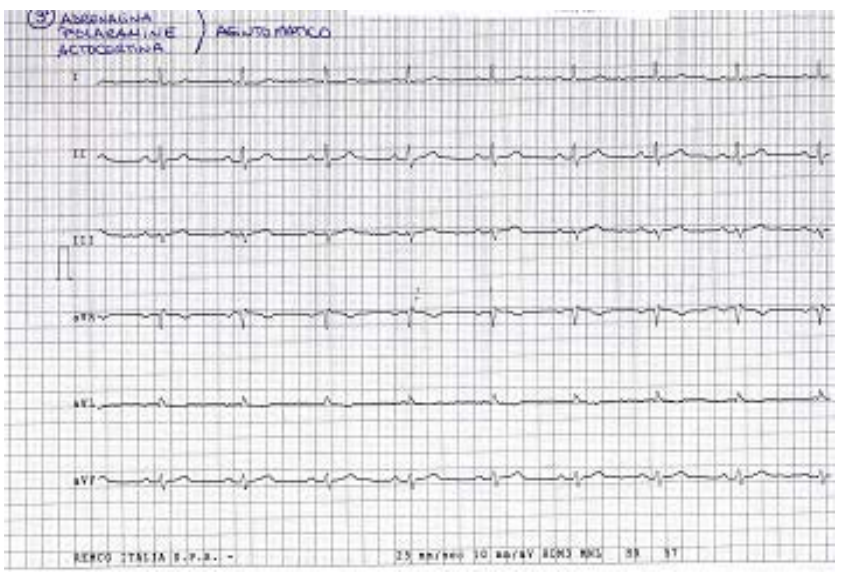




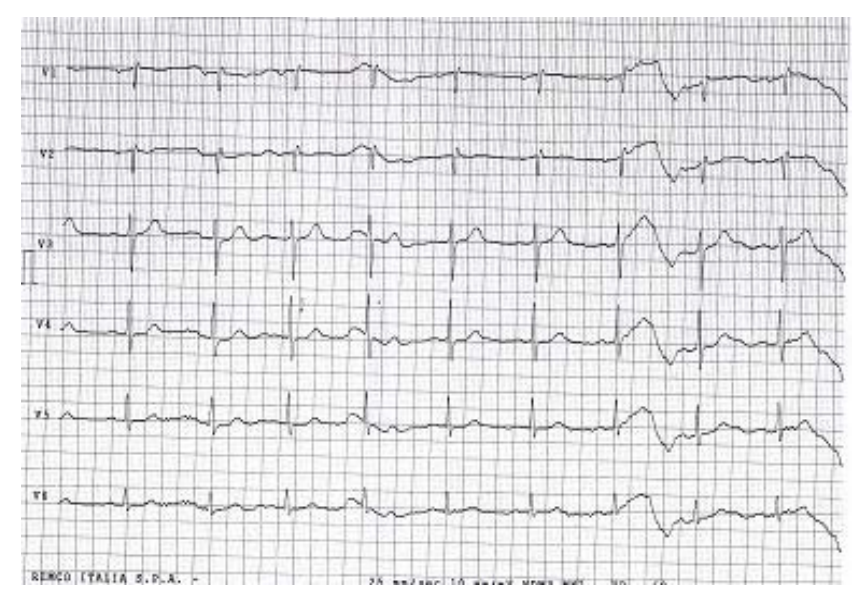

Figure 3. Coprinopsis atramentaria.

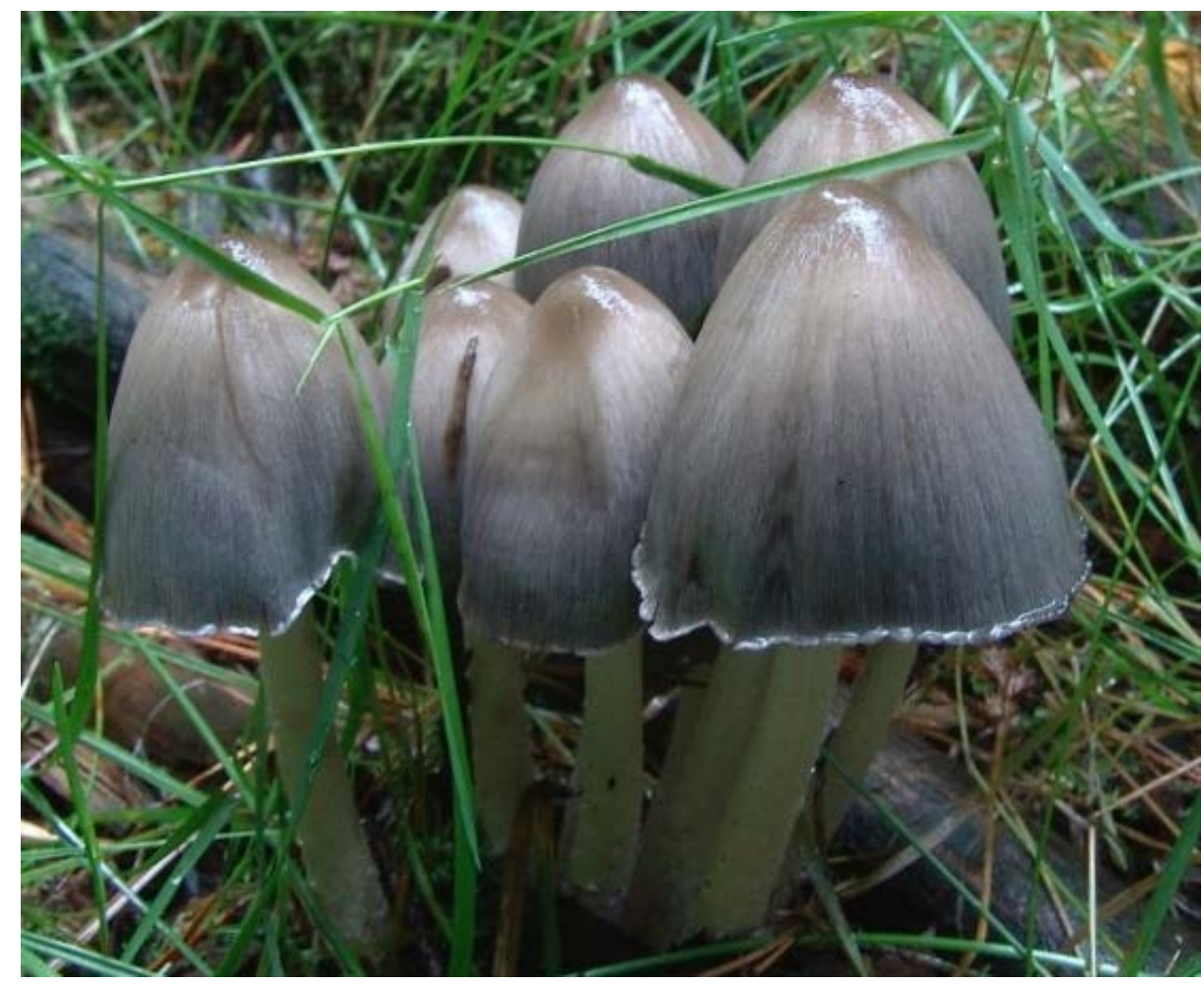

\section{References}

1. Goldfrank, LR. Mushrooms. In: Nelson LS, Lewin NA, Howland MA, Hoffman RS, Goldfrank LR, Flomenbaum NE, editor. Goldfrank's Toxicologic Emergencies. 9th ed. New York: McGraw Hill; 2010. p. 1522.

2. Bronstein AC, Spyker DA, Cantilena LR Jr, Green JL, Rumack BH, Giffin SL. 2008 Annual Report of the American Association of Poison Control Centers' National 
Poison Data System (NPDS): 26th Annual Report. Clin Toxicol (Phila) 2009;47:911.

3. Brent, J, Palmer, RB. Mushrooms. In: Shannon, MW, Borron, SW, Burns MJ, editors. Haddad and Winchester's Clinical Management of Poisoning and Drug Overdose. 4th ed. Philadelphia (PA): Saunders Elsevier; 2007. p. 455.

4. Michelot D. Poisoning by Coprinus atramentarius. Nat Toxins 1992;1:73.

5. Haberl B, Pfab R, Berndt S, Greifenhagen C, Zilker T. Case series: Alcohol intolerance with Coprine-like syndrome after consumption of the mushroom Lepiota aspera (Pers.:Fr.) Quél., 1886 (Freckled Dapperling). Clin Toxicol (Phila) 2011;49:113.

6. Pfister CW, Plice SG. Acute myocardial infarction during a prolonged allergic reaction to penicillin. Am Heart J 1950;40:945-7.

7. Kounis NG, Zavras GM. Histamine-induced coronary artery spasm: the concept of allergic angina. Br J Clin Pract 1991;45:121-8.

8. Kounis NG, Zavras GM. Allergic angina and allergic myocardial infarction. Circulation 1996;94:1789.

9. Yanagawa Y, Nishi K, Tomiharu N, Kawaguchi T. A case of takotsubo cardiomyopathy associated with Kounis syndrome. Int J Cardiol 2009;132:65-7.

10. Kounis GN, Kounis SA, Hahalis G, Kounis NG. Coronary artery spasm associated with eosinophilia: another manifestation of Kounis syndrome? Heart Lung Circ 2009;18:163-4.

11. Nikolaidis LA, Kounis NG, Grandman AH. Allergic angina and allergic myocardial infarction: a new twist on an old syndrome. Can J Cardiol 2002;18:50811.

12. Biteker M. A new classification of Kounis syndrome. Int J Cardiol 2010;145:553.

13. Cevik C, Nugent K, Shome GP, Kounis NG. Treatment of Kounis syndrome. Int J Cardiol 2010;143(3):223-6.

Joaquín Valle Alonso, Elisa Lopera Lopera, Yelda Hernández Montes, Pedro Ceballos García, José Tejeda Department of Emergency Medicine, Hospital Valle de los Pedroches, Pozoblanco, Córdoba, Spain Carmen Navarro

Department of Cardiology, Hospital Valle de los pedroches, Pozoblanco, Córdoba, Spain Corresponding author:

Joaquín Valle Alonso

Department of Emergency Medicine

Southport and Ormskirk Hospital NHS Trust

Town Lane, Southport, Merseyside, PR8 6PN

E-mail: joa51274@hotmail.com 